Martha S. Linet - Michael Hauptmann •

D. Michal Freedman - Bruce H. Alexander •

Jeremy Miller • Alice J. Sigurdson •

Michele Morin Doody

\title{
Interventional radiography and mortality risks in U.S. radiologic technologists
}

Published online: 22 July 2006

C) Springer-Verlag 2006

\begin{abstract}
With the exponential increase in minimally invasive fluoroscopically guided interventional radiologic procedures, concern has increased about the health effects on staff and patients of radiation exposure from these procedures. There has been no systematic epidemiologic investigation to quantify serious disease
\end{abstract}

This research was supported in part by contracts NO1-CP-15673, NO1-CP-51016, and NO2-CP-81121 with the National Cancer Institute, National Institutes of Health, U.S. Public Health Service. This work was conducted by researchers in the Intramural Research Program of the National Cancer Institute, National Institutes of Health.

M. S. Linet · D. M. Freedman · A. J. Sigurdson · M. M. Doody

Radiation Epidemiology Branch,

Division of Cancer Epidemiology and Genetics,

National Cancer Institute,

Bethesda, MD, USA

\section{Hauptmann}

Biostatistics Branch,

Division of Cancer Epidemiology and Genetics,

National Cancer Institute,

Bethesda, MD, USA

B. H. Alexander

Division of Environmental Health Sciences,

University of Minnesota,

Minneapolis, MN, USA

J. Miller

Information Management Services, Inc.,

Rockville, MD, USA

M. S. Linet $(\bowtie)$

Radiation Epidemiology Branch,

Division of Cancer Epidemiology and Genetics,

National Cancer Institute,

6120 Executive Blvd., EPS Room 7048,

Bethesda, MD 20892-7238, USA

e-mail: linetm@mail.nih.gov

Tel.: +1-301-4966600

Fax: +1-301-4020207 risks or mortality. To quantify all-cause, circulatory system disease and cancer mortality risks in U.S. radiologic technologists who work with interventional radiographic procedures, we evaluated mortality risks in a nationwide cohort of 88,766 U.S. radiologic technologists $(77 \%$ female $)$ who completed a self-administered questionnaire during 1994-1998 and were followed through 31 December 2003. We obtained information on work experience, types of procedures (including fluoroscopically guided interventional procedures), and protective measures plus medical, family cancer history, lifestyle, and reproductive information. Cox proportional hazards regression models were used to compute relative risks (RRs) with 95\% confidence intervals (CIs). Between completion of the questionnaire and the end of follow-up, there were 3,581 deaths, including 1,209 from malignancies and 979 from circulatory system diseases. Compared to radiologic technologists who never or rarely performed or assisted with fluoroscopically guided interventional procedures, all-cause mortality risks were not increased among those working on such procedures daily. Similarly, there was no increased risk of mortality resulting from all circulatory system diseases combined, all cancers combined, or female breast cancer among technologists who daily performed or assisted with fluoroscopically guided interventional procedures. Based on small numbers of deaths $(n=151)$, there were non-significant excesses $(40 \%-70 \%)$ in mortality from cerebrovascular disease among technologists ever working with these procedures. The absence of significantly elevated mortality risks in radiologic technologists reporting the highest frequency of interventional radiography procedures must be interpreted cautiously in light of the small number of deaths during the relatively short follow-up. The present study cannot rule out increased risks of cerebrovascular disease, specific cancers, and diseases with low case-fatality rates or a long latency period preceding death.

Keywords Radiologic technologists - Interventional radiography $\cdot$ Occupational radiation exposure $\cdot$ Mortality 


\section{Introduction}

Although the development [1] and growing use of cardiac angiography [2] revolutionized diagnostic assessment of coronary arteriosclerosis, fluoroscopy was limited mostly to examinations of the gastrointestinal tract and diagnostic angiography until the 1980s. Since then, fluoroscopy has been increasingly used to guide complex cardiac and other vascular procedures. The increasing availability of a highlevel-control fluoroscopy mode (with greatly improved visualization of detail made possible by boosting the radiation output from the X-ray tube) and other technologic advances led to exponential growth of minimally invasive interventional radiologic procedures during the 1990s. Dramatic expansion in the types, complexity, time required, and wide range of specialists performing fluoroscopically guided interventional radiologic procedures (most specialists, excluding radiologists, without appropriate training in radiation sciences) occurred concomitantly, with increasing reports of severe skin burns at X-ray beam entry sites [3, 4]. Advisories by the U.S. Food and Drug Administration [5-7] about prolonged fluoroscopy time, skin injuries, and the absence of patient dose information spawned clinical reviews of skin injuries [4] and a large survey measuring patient radiation doses from 21 types of fluoroscopically guided interventional procedures [8].

The substantial skin doses [8], unclear skin injury threshold [9], potential for treatments to require multiple interventional procedures, and preliminary data linking specific medical conditions or genetic disorders with increased radiation sensitivity $[4,10,11]$ suggest the critical need to record cumulative radiation dose and skin dose in patients' medical records [3]. Although these medical problems and associated recommendations are clearly important for patient care, clinicians have focused exclusively on preventing acute or short-term effects. The medical literature provides sparse information about organ doses or late effects associated with fluoroscopically guided interventional procedures.

Medical radiation workers, the earliest occupational group exposed to ionizing radiation [12], comprise approximately half of the radiation workers in the U.S. and 2.3 million internationally [13]. Early radiologists experienced elevated risks of leukemia, skin cancer and other radiation-related malignancies [14-18], but cancer (except possibly multiple myeloma) was not increased in radiologists first employed after 1940 [17, 18]. Studies of radiologic technologists have been inconsistent, with some [19-21] but not others [22, 23] demonstrating elevated leukemia risks. Early radiologic technologists had increased risks of basal cell, but not squamous cell, carcinoma [24], while Chinese X-ray workers experienced modest skin cancer excesses (histologic types not specified), primarily at sites of radiation-related chronic dermatitis [20]. Early, but generally not more recent, U.S. and Chinese radiologic technologists had significant female breast cancer excesses [20, 25-28]; breast cancer was not increased among Danish medical radiation workers [23].
Although radiation-related carcinogenic effects have been established for decades, reports linking elevated heart disease risks with occupational radiation exposures of early radiologists [29] and radiotherapy given to Hodgkin lymphoma patients [30] first appeared 15-20 years ago. The early radiologists and Hodgkin lymphoma patients likely experienced moderate-to-high and high-dose radiation exposures, respectively, but subsequent reports of increased heart disease and stroke were linked with substantially lower levels of radiation exposure among Japanese atomic bomb survivors [31] and U.S. radiologic technologists who first worked before 1950 [32]. The occurrence and time trends for cancer and cardiovascular disease risks in these radiation-exposed populations are consistent, with notable reduction in the recommended exposure limits from an annual level of $3 \mathrm{~Sv}$ in 1902 to 0.7 Sv in the mid-1920s, 0.3 Sv in 1934, 0.15 Sv in 1949, $0.05 \mathrm{~Sv}$ in 1957 , and $0.02 \mathrm{~Sv}$ as a 5 -year average (not to exceed $0.05 \mathrm{~Sv}$ in any single year) in 1990 [33-35].

In our follow-up of a nationwide, predominantly female cohort of U.S. radiologic technologists, we have previously described mortality and cancer incidence risks according to employment history, work practices, and related occupational characteristics among a subset of 90,305 technologists who completed a baseline selfadministered questionnaire [36]. In the current investigation, 88,766 technologists who completed a second self-administered questionnaire that addressed interventional radiographic procedures were followed through 2003 to assess mortality risks caused by various diseases. To our knowledge, this is the first large epidemiologic study to assess mortality risks in medical staff working with interventional radiologic procedures.

\section{Materials and methods}

Study population and follow-up

The U.S. Radiologic Technologists (USRT) Study is an ongoing collaboration among investigators from the U.S. National Cancer Institute, the University of Minnesota, and the American Registry of Radiologic Technologists (ARRT). Details of the study population and follow-up have been reported elsewhere [36, 37]. In brief, 146,022 radiologic technologists were enrolled into the cohort if they were certified by the ARRT during 1923-1980 for at least 2 years and resided in the U.S. or its territories. Subjects were followed through annual recertifications with the ARRT and linkage with state, national and other databases.

A baseline or first self-administered survey, carried out in the mid-1980s, inquired about employment history, demographic, lifestyle, and other factors. A second survey, which was conducted during 1994-1998, was completed by 88,766 radiologic technologists ( $72 \%$ response rate), and is the subject of this report. The second survey obtained updated information about: demographic, anthropometric, cigarette smoking, and selected other lifestyle 
factors; work experience, practices (including fluoroscopically guided interventional procedures), and protective measures; and reproductive, medical, and family cancer history. Hereafter, we refer to the second questionnaire as "the questionnaire."

\section{Deaths}

Deaths were identified through linkage with the Social Security Administration Death Mortality File and the National Death Index. Causes of death were obtained from death certificates or National Death Index Plus. Deaths were classified using the underlying cause of death coded according to the International Classification of Diseases 9 th or 10th revisions [38]. There were 3,581 deaths from all causes, including 979 from all circulatory system conditions and 1,209 from all cancers.

\section{Data collection}

The questionnaire asked respondents whether they had worked with specific radiologic procedures and, if so, the frequency of performing or assisting with the procedure for each of three time periods: before 1980, during 1980-1989, and in 1990 or later. Within each time period, the technologist was asked to identify the frequency of conducting that procedure by category: never or rarely, monthly, weekly, or daily.

Most of the technologists were born between 1940 and $1959(79 \%)$ and were, on average, 49.0 years old at completion of the second questionnaire (Table 1). Close to $95 \%$ of the cohort was Caucasian; the average body mass index (BMI) was 25.7 (25.3 for women and 27.1 for men); approximately $13 \%$ of cohort members were current smokers (one-third were former smokers and 53\% had never smoked at the time of completing the questionnaire); and $32 \%$ drank alcohol, but less than one drink per week (22\% never drank alcohol).

\section{Statistical analysis}

Person-years of follow-up were compiled from the date of questionnaire completion to the date of death, last known vital status, or 31 December 2003, whichever occurred first. Cox proportional hazards regression models were used to compute relative risks (RRs) with $95 \%$ confidence intervals (CIs) [39], using age as the survival time variable [40] and stratifying at baseline for 5-year birth cohorts to control for secular trends. Confidence intervals and significance tests were based on the asymptotic normal distribution of the risk estimates. Risks were calculated according to whether the technologist had worked with interventional radiography procedures in each of the three time periods. Compared to technologists who never or rarely worked with interventional radiography procedures, risks were calculated for those who reported working with them monthly, weekly, or daily. All statistical tests were two-sided, and $P$-values were calculated for categorical tests of trend. To account for working with interventional procedures in more than one time period, the frequency of performing these procedures in each time period, plus other covariates (summarized above and included in the legend of Table 2), were added to a single model for each cause of death category. Because several previous analyses have shown elevated cancer mortality and incidence risks and increased circulatory system disease risks among U.S. radiologic technologists who first worked prior to 1950 (or 1940), we also added the year first worked to analyses conducted using the above-described model.

\section{Results}

Work history and mortality of the cohort

Among the 88,766 U.S. radiologic technologists (77\% female) who completed the second self-administered questionnaire, there were 630,118 person-years accumulated for those successfully followed through 31 December 2003. There was an average of 7.1 years between completion of the second questionnaire and the end of follow-up. Most of the technologists first worked with medical radiation procedures in 1960 or later $(80 \%)$, began working between ages 18 and $22(72 \%)$, and worked as a radiologic technologist for 10 or more years $(80 \%$; $45 \%$ worked for 20 or more years; Table 1). During follow-up there were 3,581 deaths, including 1,209 from malignancies (176 caused by breast cancer, results not shown) and 979 from circulatory system diseases (151 caused by cerebrovascular disease; Table 2).

Mortality risks in technologists performing or assisting with fluoroscopically guided interventional procedures

The number of technologists who reported ever performing or assisting with fluoroscopically guided interventional procedures was 17,107 before 1980 and declined in subsequent years, though the proportion of technologists who reported working daily with these procedures rose from $32 \%$ before 1980 to $37 \%$ during $1980-1989$ and $44 \%$ in 1990 or later.

All-cause mortality was not increased among radiologic technologists who ever performed or assisted with fluoroscopically guided interventional procedures compared to those who never performed such procedures (Table 2). Similarly, there was no evidence of elevated mortality risks from all circulatory system diseases or all cancers combined. Likewise, there was no excess of female breast cancer before 1980 (RR 1.2, 95\% CI 0.7-1.9), during $1980-1989$ (RR $0.8,95 \%$ CI $0.4-1.8$ ), or in 1990 or later (RR 1.0, 95\% CI 0.4-2.5). Based on small numbers of deaths $(n=151)$, there were non-significant increases $(40 \%-70 \%)$ in mortality from cerebrovascular disease in two of the three time periods among technologists who had 
Table 1 Demographic and selected work history and lifestyle characteristics by gender in U.S. radiologic technologists who completed a self-administered questionnaire during 1994-1998

\begin{tabular}{|c|c|c|c|c|}
\hline \multirow[t]{2}{*}{ Characteristics } & \multicolumn{2}{|c|}{ Females $(n=68,312)$} & \multicolumn{2}{|c|}{ Males $(n=20,454)$} \\
\hline & Number & $\%$ & Number & $\%$ \\
\hline \multicolumn{5}{|l|}{ Birth year } \\
\hline$<1930$ & 3,950 & 5.8 & 2,182 & 10.7 \\
\hline 1930-39 & 8,678 & 12.7 & 3,409 & 16.7 \\
\hline $1940-49$ & 22,278 & 32.6 & 7,271 & 35.6 \\
\hline $1950-59$ & 32,735 & 47.9 & 7,547 & 36.9 \\
\hline $1960+$ & 671 & 1.0 & 45 & 0.2 \\
\hline \multicolumn{5}{|l|}{ Age at completion of questionnaire } \\
\hline$<30$ & 0 & - & 0 & - \\
\hline $30-39$ & 12,502 & 18.3 & 1,756 & 8.6 \\
\hline $40-49$ & 32,334 & 47.3 & 9,496 & 46.4 \\
\hline $50-59$ & 15,450 & 22.6 & 5,222 & 25.5 \\
\hline $60-69$ & 5,641 & 8.3 & 2,681 & 13.1 \\
\hline$\geq 70$ & 2,385 & 3.5 & 1,299 & 6.3 \\
\hline \multicolumn{5}{|l|}{ Race } \\
\hline White & 65,515 & 95.9 & 18,499 & 90.4 \\
\hline Black & 1,789 & 2.6 & 917 & 4.5 \\
\hline Asian/Pacific Islander & 476 & 0.7 & 559 & 2.7 \\
\hline American Indian/Alaskan Native & 125 & 0.2 & 114 & 0.6 \\
\hline Other & 347 & 0.5 & 329 & 1.6 \\
\hline Unknown & 60 & 0.1 & 36 & 0.2 \\
\hline \multicolumn{5}{|l|}{ BMI } \\
\hline$<18.5$ & 1,205 & 1.8 & 110 & 0.5 \\
\hline $18.5-24.9$ & 36,898 & 54.0 & 6,036 & 29.5 \\
\hline $25.0-29.9$ & 17,864 & 26.2 & 9,828 & 48.1 \\
\hline $30.0-34.9$ & 7,164 & 10.5 & 3,124 & 15.3 \\
\hline$\geq 35.0$ & 3,636 & 5.3 & 990 & 4.8 \\
\hline Unknown & 1,545 & 2.3 & 366 & 1.8 \\
\hline \multicolumn{5}{|l|}{ Cigarette smoking } \\
\hline Never smoked & 37,935 & 55.5 & 8,893 & 43.5 \\
\hline Former smoker & 21,083 & 30.9 & 8,258 & 40.4 \\
\hline Current smoker & 8,794 & 12.9 & 3,077 & 15.0 \\
\hline Unknown if smoked & 500 & 0.7 & 226 & 1.1 \\
\hline \multicolumn{5}{|l|}{ Alcohol consumption } \\
\hline Never & 15,321 & 22.4 & 4,569 & 22.3 \\
\hline$<1$ per week & 23,377 & 34.2 & 4,745 & 23.2 \\
\hline $1-6$ per week & 17,713 & 25.9 & 5,745 & 28.1 \\
\hline 7-13 per week & 3,427 & 5.0 & 1,512 & 7.4 \\
\hline$\geq 14$ per week & 2,750 & 4.0 & 1,954 & 9.6 \\
\hline Unknown & 5,724 & 8.4 & 1,929 & 9.4 \\
\hline \multicolumn{5}{|c|}{ Year first worked with medical radiation procedures } \\
\hline$<1940$ & 511 & 0.8 & 186 & 0.9 \\
\hline $1940-49$ & 2,399 & 3.5 & 1,239 & 6.1 \\
\hline $1950-59$ & 9,671 & 14.2 & 3,156 & 15.4 \\
\hline $1960-69$ & 22,758 & 33.3 & 5,871 & 28.7 \\
\hline$\geq 1970$ & 32,505 & 47.6 & 9,845 & 48.1 \\
\hline Unknown & 468 & 0.7 & 157 & 0.8 \\
\hline \multicolumn{5}{|l|}{ Age first worked } \\
\hline$<18$ & 6,921 & 10.1 & 1,091 & 5.3 \\
\hline $18-19$ & 34,526 & 50.5 & 5,578 & 27.3 \\
\hline $20-22$ & 17,004 & 24.9 & 6,646 & 32.5 \\
\hline
\end{tabular}


Table 1 (continued)

\begin{tabular}{lcccc}
\hline Characteristics & Females $(n=68,312)$ & & \multicolumn{2}{c}{ Males $(n=20,454)$} \\
\cline { 2 - 4 } & Number & $\%$ & 5.3 & Number \\
\hline $23-24$ & 3,623 & 8.5 & 2,847 & 13.9 \\
$\geq 25$ & 5,770 & 0.7 & 4,135 & 20.2 \\
Unknown & 468 & & 157 & 0.8 \\
Total number of years worked & 13,726 & 20.1 & 3,417 & 16.7 \\
$<10$ & 25,149 & 36.8 & 6,013 & 29.4 \\
$10-19$ & 28,969 & 42.4 & 10,867 & 53.1 \\
$\geq 20$ & 468 & 0.7 & 157 & 0.8 \\
Unknown & & & \\
\hline
\end{tabular}

reported ever working with these procedures. Risks were not significantly increased in technologists who worked with these procedures on a daily basis compared to those who never or rarely performed such procedures for any of the major categories of cause of death (i.e., all-cause, circulatory system diseases, or total malignancies) or for deaths caused by cerebrovascular disease or breast cancer. In addition, there was no evidence of a significant doseresponse according to frequency of performing or assisting with fluoroscopically guided interventional procedures for mortality from all causes, all circulatory system diseases (or the subcategory of cerebrovascular disease) or total malignancies (or the subcategory of female breast cancer, data not shown) for any of the three time periods evaluated. Statistically significant 1.8 - and 3.8-fold mortality risks were observed for circulatory system and cerebrovascular diseases (based on 21 and 4 deaths, respectively), and a borderline significant 1.3-fold risk seen for all-cause mortality in technologists who reported performing interventional procedures weekly in 1990 or later. The above results did not change when the analyses were additionally adjusted for year first worked (data not shown).

Mortality risks in relation to other risk factors

In contrast to the lack of clearly and consistently elevated risks or radiation-related dose-response for any mortality outcomes in those working with fluoroscopically guided interventional procedures, significantly elevated risks were apparent for several known risk factors for the causes of death evaluated. Mortality risks were significantly higher for males than females for all-cause and circulatory system mortality; risks declined with increasing alcohol consumption for all circulatory system (including cerebrovascular) disease; and mortality risks from all causes, all circulatory system diseases, and total malignancies were approximately threefold higher among current cigarette smokers. Mortality risks were $50 \%-80 \%$ increased for those who were underweight $(<18.5 \mathrm{BMI})$ and $30 \%-90 \%$ increased for those who were obese $(\geq 35.0 \mathrm{BMI})$ for all causes, circulatory system diseases, and total malignancies, though a trend of increasing mortality with increasing BMI was statistically significant for all causes and circulatory system diseases but not total malignancies.

\section{Discussion}

Compared with technologists who reported never performing or assisting with fluoroscopically guided interventional procedures, those who indicated that they had ever worked with such procedures did not experience increased mortality from all causes of death, all circulatory system diseases (or the subcategory of cerebrovascular disease), or all malignancies combined (or female breast cancer). Evaluation of mortality risks for these major categories or specific causes of death did not reveal any significant excesses among technologists who performed or assisted with these procedures on a daily basis, though statistically significantly elevated risks were found for all circulatory system diseases and cerebrovascular disease among technologists who worked with interventional procedures weekly in 1990 or later based on small numbers of deaths. There was, however, no evidence of a pattern of increasing risk with increasing frequency of working with fluoroscopically guided interventional procedures for any of the mortality outcomes for any of the three time periods examined.

To our knowledge, this is the only large epidemiologic cohort investigation that has assessed the relation between the use of interventional radiographic procedures and mortality risks among medical staff performing or assisting with such procedures. A special feature that distinguishes our cohort from other populations of medical radiation workers is the large subpopulation of female medical radiation workers exposed to protracted, primarily lowdose ionizing radiation. The large size of the study population provides the opportunity to make internal comparisons and, thus, minimize potential biases resulting from the healthy worker effect. The cohort is also unique in its nationwide coverage, the nearly complete mortality follow-up, and the availability of data on other risk factors that might confound mortality risks from all causes, all circulatory system diseases, cerebrovascular disease, all malignancies, and breast cancer. The extensive data collection on risk factors other than work-related data enabled us to adjust for potential confounding effects. Our study also collected data on work history, a wide range of radiologic procedures, behavioral characteristics, and protective measures. Another key feature of the study was the low proportion of missing data. 


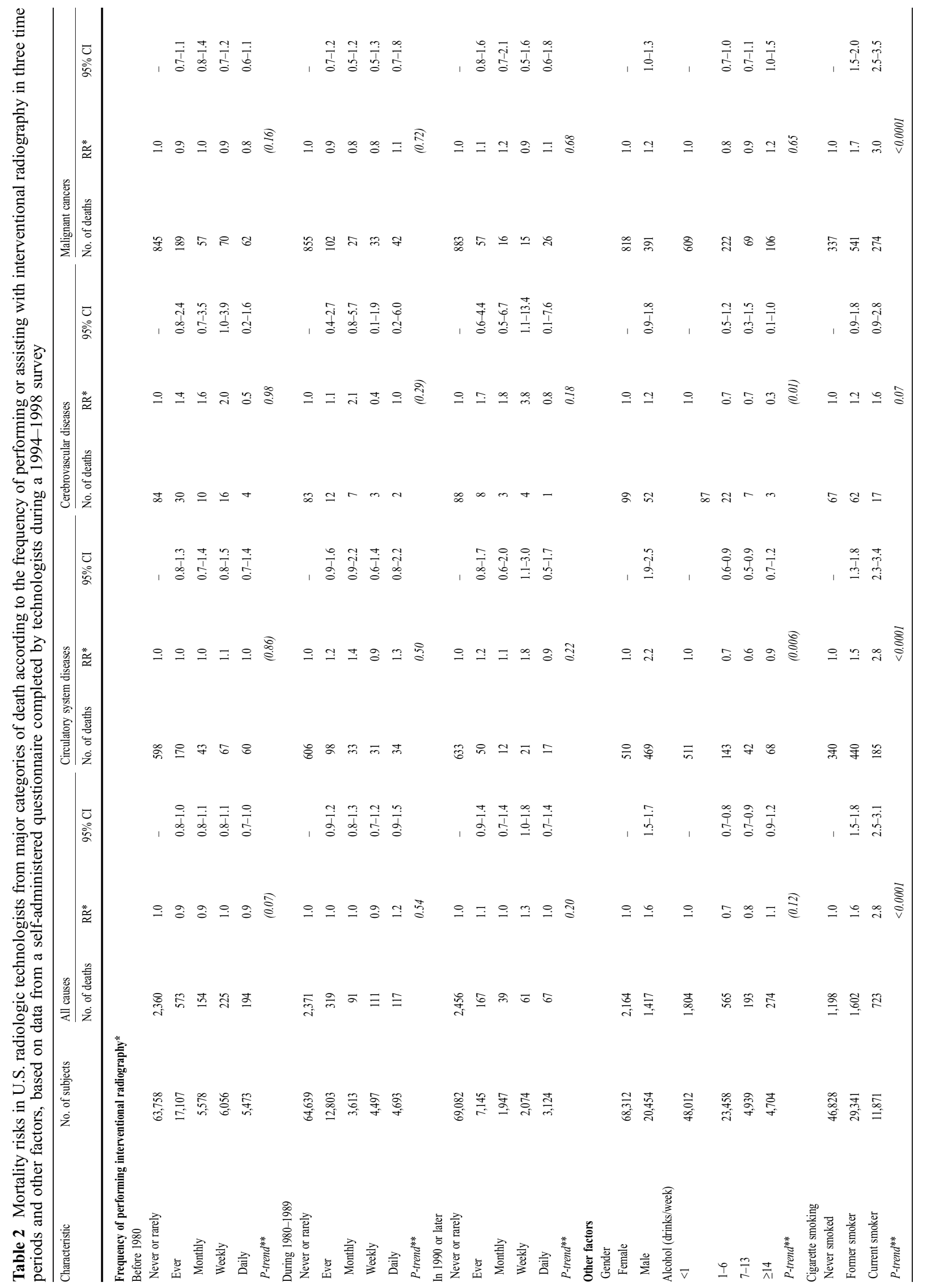




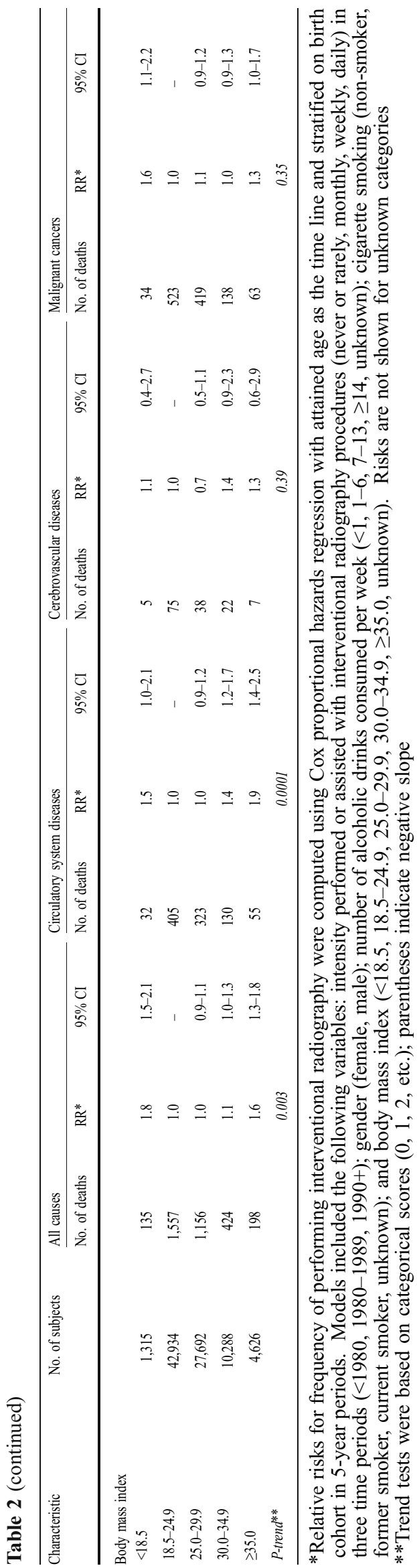

The main limitations of our study are the relatively short follow-up period for the technologists subsequent to completion of the questionnaire and the absence of individual lifetime occupational radiation dosimetry data. Our analysis focused on surrogate measures for performing or assisting with fluoroscopically guided interventional procedures based on responses to the self-administered questionnaire. The subset of questionnaire respondents might not be representative of all radiologic technologists in the cohort because respondents had to survive until the mid-1990s and complete the questionnaire.

The fact that we did not observe clear patterns of excess mortality risks at this time does not mean that working with fluoroscopically guided interventional radiographic procedures is safe. Because these procedures came into common usage only in the early 1980 s, the time since first exposure is relatively short (i.e., $<20$ years) for most technologists, and follow-up in this study was only about 7 years on average. Furthermore, the U.S. Radiologic Technologists cohort was still young at the end of follow-up (mean age was 56 years), ages when mortality is low in general, especially from circulatory system diseases and malignancies. We will continue to follow the cohort, including the subset of technologists performing or assisting with interventional radiography exposures, and we anticipate that more definitive mortality risk estimates will be forthcoming as additional follow-up accrues.

Acknowledgements We are grateful to the radiologic technologists who participated in the cohort study of U.S. Radiologic Technologists, Jerry Reid of the American Registry of Radiologic Technologists for continued support of this research project, Diane Kampa of the University of Minnesota for study management, and Chris McClure of Research Triangle Institute for assessment of mortality outcomes. We also thank Drs. John Boice and Jack Mandel, who played key roles in establishing the epidemiologic follow-up study of U.S. radiologic technologists.

\section{References}

1. Sabiston DC Jr (1974) The William F. Rienhoff Jr. lecture. The coronary circulation. Johns Hopkins Med J 134:314-329

2. Riley RC, Birks JW, Palacios E, et al (1972) Exposure of radiologists during special procedures. Radiology 104:679-683

3. Marx MV (2003) The radiation dose in interventional radiology study: knowledge brings responsibility. J Vasc Interv Radiol 14:947-951

4. Wagner LK, McNeese MD, Marx MV, et al (1999) Severe skin reactions from interventional fluoroscopy: case report and review of the literature. Radiology 21:773-776

5. Food and Drug Administration (1994) Important information for physicians and other health care professionals: avoidance of serious $x$-ray induced skin injuries to patients during fluoroscopically-guided procedures. Center for Devices and Radiological Health, Rockville, Md., 9 September. http://www.fda. gov/cdrh/fluor.html

6. Food and Drug Administration (1994) FDA Public Health Advisory: Avoidance of serious x-ray induced skin injuries to patients during fluoroscopically-guided procedures. Center for Devices and Radiological Health, Rockville, Md., 30 September. http://www.fda.gov/cdrh/fluor.html 
7. Food and Drug Administration (1995) Recording information in the patient's medical record that identifies the potential for serious X-ray induced skin injuries. Center for Devices and Radiological Health, Rockville, Md., 15 September. http://www.fda.gov/cdrh/xrayinj.html

8. Miller DL, Balter S, Cole PE, et al (2003) Radiation doses in interventional radiology procedures: the RAD-IR study. II. Skin dose. J Vasc Interv Radiol 14:711-727

9. Mettler FA Jr, Voelz GL (2002) Major radiation exposure what to expect and how to respond. N Engl J Med 346:15541561

10. Morris MM, Powell SN (1997) Irradiation in the setting of collagen vascular disease: acute and late complications. J Clin Oncol 15:2728-2735

11. Herold DM, Hanlon AL, Hanks GE (1999) Diabetes mellitus: a predictor for late radiation morbidity. Int J Radiat Oncol Biol Phys 43:475-479

12. Kumazawa S, Nelson DR, Richardson ACB (1992) Occupational exposure to ionizing radiation in the United States: a comprehensive review for the years 1960-1985. In: United States Environmental Protection Agency, EPA52011-84-005. Washington, DC, p 100

13. International Commission on Radiological Protection (2000) Avoidance of radiation injuries from medical interventional procedures. ICRP Publication 85. Ann ICRP 30:7-67

14. Smith PG, Doll R (1981) Mortality from cancer and all causes among British radiologists. Br J Radiol 54:187-194

15. Berrington A, Darby SC, Weiss HA, et al (2001) 100 years of observation on British radiologists: mortality from cancer and other causes 1987-1997. Br J Radiol 74:507-519

16. Ulrich $H$ (1946) The incidence of leukemia in radiologists. N Engl J Med 234:45-46

17. Lewis EB (1963) Leukemia, multiple myeloma, and aplastic anemia in American radiologists. Science 142:1492-1494

18. Matanoski GM, Seltser R, Sartwell PE, et al (1975) The current mortality rates of radiologists and other physician specialists: specific causes of death. Am J Epidemiol 101:199-210

19. Yoshinaga S, Aoyama T, Yoshimoto Y, et al (1999) Cancer mortality among radiological technologists in Japan: updated analysis of follow-up data from 1969-1993. J Epidemiol 9:61-72

20. Wang JX, Zhang LA, Li BX, et al (2002) Cancer incidence and risk estimation among medical x-ray workers in China, 19501995. Health Phys 82:455-466

21. Linet MS, Freedman DM, Mohan AK, et al (2005) Incidence of haematopoietic malignancies in U.S. radiologic technologists. Occup Environ Med 62:861-867

22. Jablon S, Miller RW (1978) Army technologists: 29-year follow-up for cause of death. Radiology 126:677-679

23. Andersson M, Engholm G, Ennow K, et al (1991) Cancer risk among staff at two radiotherapy departments in Denmark. Br J Radiol 64:455-460
24. Yoshinaga S, Hauptmann M, Sigurdson AJ, et al (2005) Nonmelanoma skin cancer in relation to ionizing radiation exposure among U.S. radiologic technologists. Int J Cancer 115:828-834

25. Wang JX, Inskip PD, Boice JD Jr, et al (1990) Cancer incidence among medical diagnostic $\mathrm{x}$-ray workers in China, 1950 to 1985. Int J Cancer 45:889-895

26. Mohan AK, Hauptmann M, Linet MS, et al (2002) Breast cancer mortality among female radiologic technologists in the United States. J Natl Cancer Inst 94:943-948

27. Sigurdson AJ, Doody MM, Rao RS, et al (2003) Cancer incidence in the U.S. Radiologic Technologists Health Study, 1983-1998. Cancer 97:3080-3089

28. Doody MM, Freedman DM, Alexander BH, et al (2006) Breast cancer incidence in U.S. radiologic technologists. Cancer, DOI $10.1002 /$ cncr.21876

29. Matanoski GM, Sartwell P, Elliot E, et al (1984) Cancer risk in radiologists and radiation workers. In: Boice JD Jr, Fraumeni JF $\mathrm{Jr}$ (eds) Radiation carcinogenesis: epidemiology and biological significance. Raven Press, New York, pp 83-96

30. Hancock SL, Tucker MA, Hoppe RT (1993) Factors affecting late mortality from heart disease after treatment of Hodgkin's disease. JAMA 270:1949-1955

31. Shimizu Y, Pierce DA, Preston DL, et al (1999) Studies of the mortality of atomic bomb survivors. Report 12, part II. Noncancer mortality: 1950-1990. Radiat Res 152:374-389

32. Hauptmann M, Mohan AK, Doody MM, et al (2003) Mortality from diseases of the circulatory system in radiologic technologists in the United States. Am J Epidemiol 157:239-248

33. International Commission on Radiological Protection (1991) 1990 Recommendation. Pergamon Press, Oxford, UK

34. Inkret WC, Meinhold CB, Taschner JC (1995) Radiation and risk - a hard look at the data. Protection Standards. Los Alamos Science 23:117-124

35. Brodsky A, Kathren RL, Willis CA (1995) History of the medical uses of radiation: regulatory and voluntary standards of protection. Health Phys 69:783-823

36. Boice JD Jr, Mandel JS, Doody MM, et al (1992) A health survey of radiologic technologists. Cancer 69:586-598

37. Mohan AK, Hauptmann M, Freedman DM, et al (2003) Cancer and other causes of mortality among radiologic technologists in the United States. Int J Cancer 103:259-267

38. International classification of diseases, 9th revision (1999) Practice Management Information Corp., Los Angeles, Calif

39. Kalbfleisch JD, Prentice RL (2002) The statistical analysis of failure time data. In: Probability and statistics, 2nd edn. Wiley, New York

40. Korn EL, Graubard BI, Midthune D (1997) Time-to-event analysis of longitudinal follow-up of a survey: choice of the time-scale. Am J Epidemiol 145:72-80 